\title{
Insulin receptor endocytosis in the pathophysiology of insulin resistance
}

\author{
Catherine Hall', Hongtao $\mathrm{Yu}^{2,3}$ and Eunhee Choi (id)
}

\begin{abstract}
Insulin signaling controls cell growth and metabolic homeostasis. Dysregulation of this pathway causes metabolic diseases such as diabetes. Insulin signaling pathways have been extensively studied. Upon insulin binding, the insulin receptor (IR) triggers downstream signaling cascades. The active IR is then internalized by clathrin-mediated endocytosis. Despite decades of studies, the mechanism and regulation of clathrin-mediated endocytosis of IR remain incompletely understood. Recent studies have revealed feedback regulation of IR endocytosis through Src homology phosphatase 2 (SHP2) and the mitogen-activated protein kinase (MAPK) pathway. Here we review the molecular mechanism of IR endocytosis and its impact on the pathophysiology of insulin resistance, and discuss the potential of SHP2 as a therapeutic target for type 2 diabetes.
\end{abstract}

\section{Introduction}

The pancreatic hormone insulin controls the metabolism of glucose and lipids in our body ${ }^{1,2}$. It promotes glucose uptake and its conversion into glycogen and lipids for energy storage in metabolic tissues, thereby enabling the maintenance of proper blood glucose levels. Normal circulating insulin levels are necessary for glucose homeostasis. Persistent hyperinsulinemia, an above normal level of insulin in the blood, is associated with insulin resistance. Insulin resistance is a hallmark of metabolic diseases, including type 2 diabetes and atherosclerosis ${ }^{1-6}$. Understanding the mechanisms of insulin resistance is therefore essential for the continued development of effective therapeutic strategies to treat these prevalent diseases.

The relationship between hyperinsulinemia and insulin resistance is complicated. The prevailing view is that the pancreas produces more insulin to compensate for the

Correspondence: Hongtao Yu (Hongtao.Yu@UTsouthwestern.edu) or Eunhee Choi (EC3477@cumc.columbia.edu)

${ }^{1}$ Department of Pathology and Cell Biology, Vagelos College of Physicians and Surgeons, Columbia University, 630 West 168th Street, New York, NY 10032, USA

'Laboratory of Cell Biology, School of Life Sciences, Westlake University, Hangzhou, Zhejiang 310024, China

Full list of author information is available at the end of the article rise in blood glucose level caused by defective insulin signaling ${ }^{7-10}$. An alternative view is that hyperinsulinemia may initiate and expand insulin resistance ${ }^{11-14}$. These are not mutually exclusive concepts and probably act in parallel.

At the cellular level, insulin binds to the insulin receptor (IR) on the plasma membrane (PM) and triggers the activation of signaling cascades to regulate metabolism and cell growth. Following activation, insulin-bound IR can be internalized by clathrin-mediated endocytosis $(\mathrm{CME})^{15-18}$. As a key CME adaptor, the assembly polypeptide 2 (AP2) complex links clathrin to both the cargo and lipids on the PM. The AP2 complex has four subunits: AP2A, AP2B1, AP2M1, and AP2S1. It has a large globular core consisting of the entirety of both AP2M1 and AP2S1 subunits, along with the $\mathrm{N}$-terminal trunk domain of AP2A and AP2B1. The AP2 core recognizes sorting signals from the cargo, such as di-leucine and YXXФ (X, any amino acids; $\Phi$, hydrophobic residues) motifs. The C-terminal appendages of the AP2A and AP2B1 subunits extend from the core and bind to clathrin and other accessory proteins, thus promoting clathrin vesicle formation. The endocytosis of the IR-insulin complex is a key mechanism that regulates the intensity and duration of insulin signaling. In contrast, persistent 
hyperinsulinemia may accelerate IR endocytosis, thus decreasing the functional IR level at the PM. Biochemical and immunohistochemistry studies have shown that the level of IR at the PM might be reduced in diabetes patients $^{19-21}$. These findings suggest that reduced IR levels at the PM might be a contributing factor to insulin resistance in human patients.

The spindle checkpoint ensures accurate chromosome segregation and prevents aneuploidy ${ }^{16,22-27}$. MAD2 and BUBR1 are critical spindle checkpoint proteins. In response to unattached kinetochores, they bind to $\mathrm{CDC} 20$ and BUB3 to form the mitotic checkpoint complex (MCC). MCC prevents chromosome segregation by directly inhibiting the anaphase-promoting complex/ cyclosome ${ }^{28-34}$. When all kinetochores are attached to the bipolar spindle, $\mathrm{p} 31^{\text {comet }}$ binds to active MAD2 and inactivates the spindle checkpoint ${ }^{35-39}$.

Our previous studies have revealed an unexpected function of the spindle checkpoint in insulin signaling through regulating IR endocytosis. MAD2 binds to the Cterminal MAD2-interacting motifs (MIMs) of IR and recruits AP2B1 to IR through BUBR1-CDC20 $0^{16,21,40}$ (Fig. $1 \mathrm{a}, \mathrm{b}) . \mathrm{p} 31^{\text {comet }}$ prevents IR endocytosis by inhibiting the interaction between BUBR1-CDC20-AP2 and IR-bound MAD2. Liver-specific $p 31^{\text {comet-/- }}$ mice have reduced IR levels on the PM of hepatocytes and develop whole-body insulin resistance ${ }^{40}$. Conversely, BUBR1 deficiency delays insulin-mediated IR endocytosis and improves insulin sensitivity in mice ${ }^{40,41}$. These findings suggest that the dysregulation of IR endocytosis is a potential mechanism underlying insulin resistance.

It has long been known that IR kinase activity is crucial for receptor endocytosis ${ }^{42,43}$, suggesting that IR endocytosis normally occurs after the receptor has been activated and has transduced signals downstream. However, how activated IR is selectively internalized remained largely unknown until our recent study. We have discovered a regulatory feedback mechanism of IR endocytosis through the SHP2-MAPK pathway ${ }^{21}$. Inhibition of this regulatory feedback delays IR endocytosis, prolongs metabolic signaling, and improves insulin sensitivity. Here we review this newly discovered regulatory mechanism of IR endocytosis, discuss its impact on pathophysiology, and highlight the key unanswered questions.

\section{The SHP2-MAPK pathway in metabolic regulation}

IR is a receptor tyrosine kinase (RTK) that is activated by insulin binding ${ }^{44}$. The binding of multiple insulin molecules to an IR destabilizes its autoinhibitory conformation, leading to its trans-autophosphorylation and activation ${ }^{45-47}$. The tyrosine-phosphorylated IR recruits and phosphorylates IR substrate (IRS) or SRC homology 2 domain-containing (SHC) proteins at several tyrosine residues $^{48,49}$ (Fig. 1a). These tyrosine phosphorylation events recruit additional effectors and activate two major signaling cascades: (1) the phosphatidylinositol 3kinase (PI3K)-protein kinase B/AKT (PI3K-PKB/AKT) pathway and (2) the MAPK pathway. The PI3K-PKB/ AKT pathway is primarily responsible for controlling metabolism. The MAPK pathway mainly controls cell growth and proliferation. Accumulating evidence now suggests that the dysregulation of insulin-mediated MAPK pathway activation may contribute to insulin resistance ${ }^{50-53}$.

The phosphorylated IRS and SHC proteins bind to growth factor receptor-bound protein 2 (GRB2) and then recruit the guanine nucleotide exchange factor, son of sevenless (SOS), to activate the RAS-MAPK pathway ${ }^{54}$. SHP2, encoded by PTPN11, is a nonreceptor protein tyrosine phosphatase (PTP) and a scaffolding protein that controls SOS2-mediated MAPK pathway activation. SHP2 contains two tandem $\mathrm{SH} 2$ domains (N-terminal SH2 and C-terminal SH2), a PTP domain, and a C-terminal tail ${ }^{55}$ (Fig. 1c). In the basal state, the $\mathrm{SH} 2$ domains engage the catalytic pocket in the PTP domain and sterically block the active site. Upon insulin stimulation, the two SH2 domains in SHP2 interact with the phosphotyrosine sites in IRS proteins and GRB2-associated binder protein 1 (GAB1), thus breaking the autoinhibitory interface and rendering the active site available for substrates ${ }^{56,57}$. The phosphatase activity of SHP2 is required for the formation of the GAB1-GRB2-SOS1 complex, which in turn promotes RAS activation ${ }^{58}$ (Fig. 1a). Activated RAS binds to RAF and causes RAF translocation to the PM. RAF then activates the dual-specificity serine and threonine kinases, MEK1 and MEK2, which phosphorylate and activate extracellular signal-regulated kinase 1 and 2 (ERK1 and ERK2 $)^{59}$.

ERK1/2 are the best-characterized MAPK family members. ERK1/2 phosphorylate serine or threonine residues that are followed by a proline residue (S/T-P). Over 200 substrates of ERK1/2, including SREBP1, SREBP2 ${ }^{60,61}$, and peroxisome proliferator-activated receptor $\gamma^{52}$, have been identified so far $^{62-64}$. ERK1 and ERK2 share $75 \%$ amino acid identity and phosphorylate the same substrates with similar specificity in vitro. However, $E R K 1^{-1-}$ mice are viable and fertile, but $E R K 2^{-1-}$ mice are not viable ${ }^{65-68}$, suggesting that these kinases are not redundant and have tissue-specific roles. Although $E R K 1^{-1-}$ mice have been shown to be more sensitive to insulin, diet-induced obesity mice and leptin-deficient $(o b / o b)$ mice have elevated ERK activity ${ }^{51,69,70}$. Pharmacological inhibition of ERK improves insulin sensitivity in both diet-induced obesity and $o b / o b$ mice $^{52}$. Furthermore, the basal activity of ERK is elevated in human type 2 diabetes $^{71-73}$, indicating that the MAPK pathway may be a potential therapeutic target for insulin resistance and metabolic disorders. 


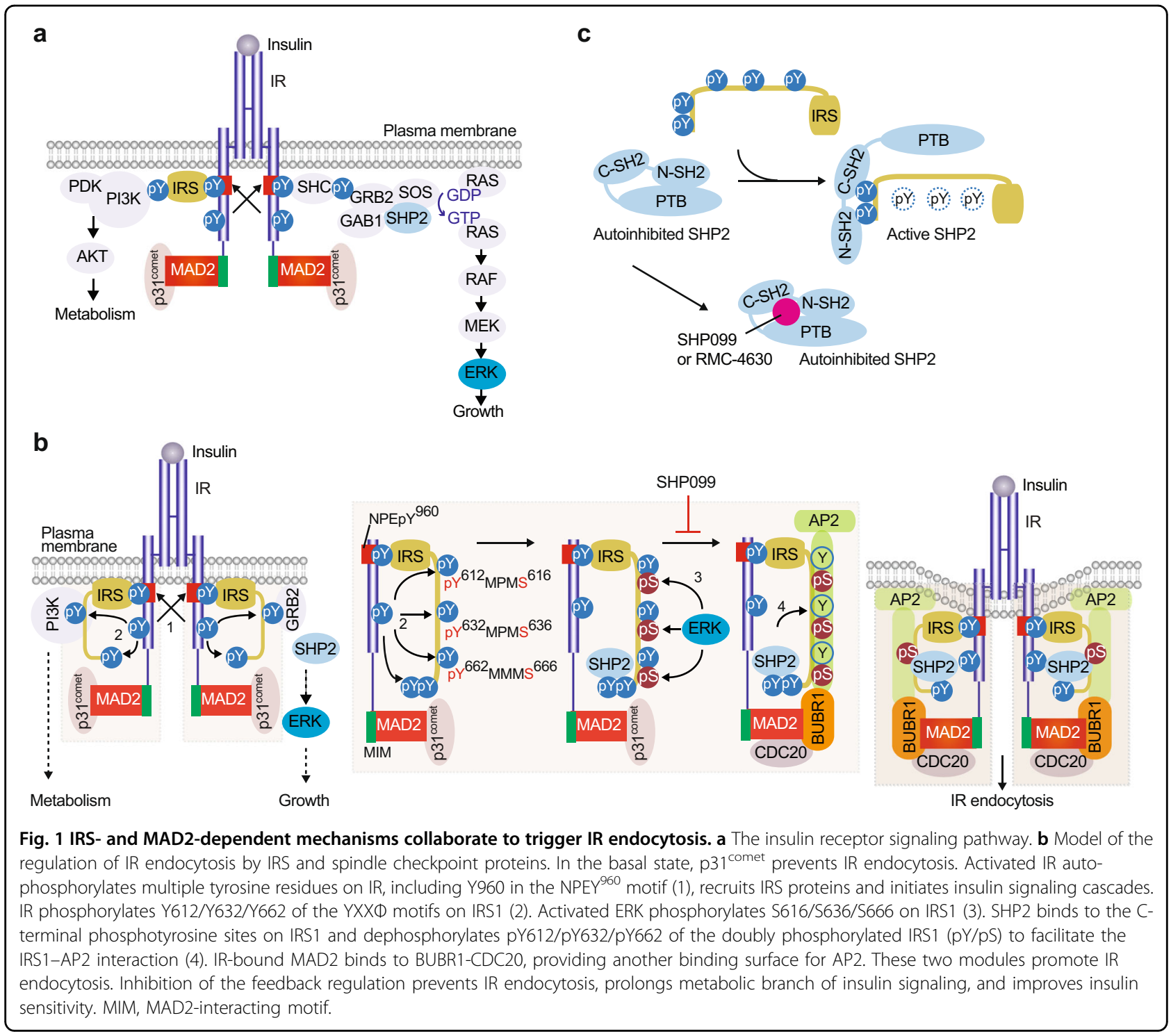

SHP2 is the first reported oncogenic tyrosine phosphatase. As an upstream regulator of the MAPK pathway, SHP2 promotes cell growth and proliferation. Conventional $S H P 2^{-1-}$ mice are embryonic lethal ${ }^{74}$. Tissuespecific $S H P 2^{-1-}$ mice survive and show that SHP2 controls metabolic homeostasis in multiple tissues. For example, striated and cardiac muscle-specific $S H P 2^{-/-}$ mice display severe dilated cardiomyopathy, undergo premature death, and exhibit insulin resistance ${ }^{75}$. Neuronal SHP2 dysfunction causes early-onset obesity accompanied by high levels of leptin, insulin, glucose, and triglycerides $^{76}$. On the other hand, liver-specific $S H P 2^{-/-}$ mice exhibit enhanced insulin sensitivity ${ }^{77,78}$. Pharmacological inhibition of SHP2 markedly increased glucose and insulin sensitivity in a diet-induced obesity mouse model $^{21}$. The introduction of adeno-associated viruses encoding SHP2 short-hairpin RNAs into the liver confirms a role of SHP2 in metabolic homeostasis in mice $^{21}$. In addition, deficiency of GAB1, the binding partner of SHP2, in the liver exhibits improved insulin sensitivity, together with enhanced AKT and blunted MAPK pathway activation ${ }^{79}$. This finding suggests that the SHP2-MAPK pathway may offset certain aspects of insulin signaling in the liver, thus prolonging the metabolism signaling branch and improving whole-body insulin sensitivity.

\section{The SHP2-MAPK pathway in IR endocytosis}

How does the SHP2-MAPK pathway control metabolism? What are the main targets of SHP2 and MAPK in this pathway? IRS proteins are crucial adaptors that transduce signals from IR on the PM to intracellular downstream effectors and adaptors ${ }^{48}$. Insulin-activated IR phosphorylates its own $\mathrm{NPEY}^{960}$ motif in the 

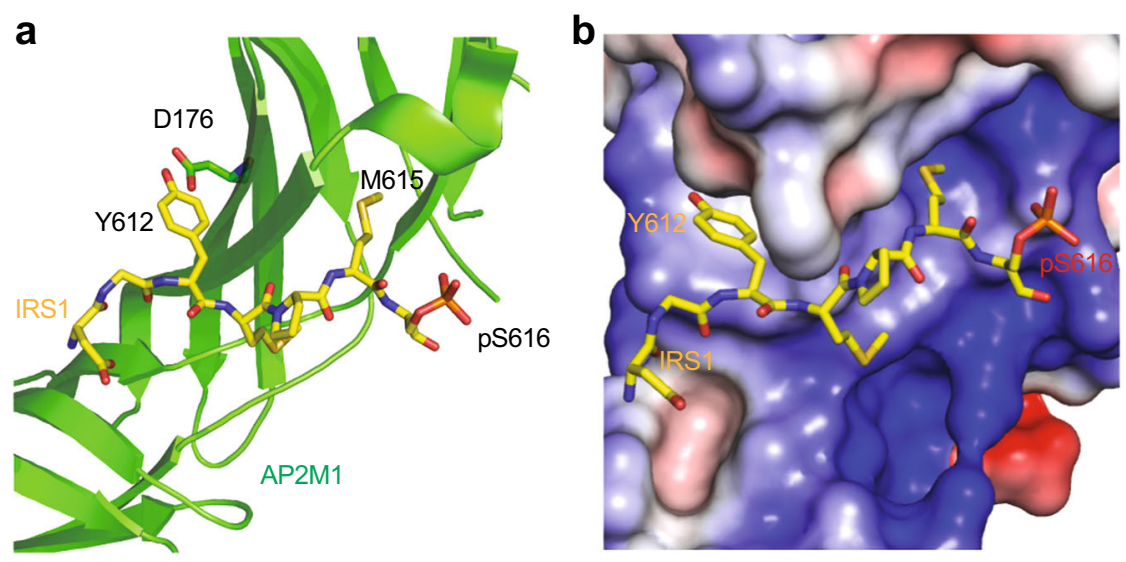

Fig. 2 Structure of AP2M1 bound to pS616-IRS1. a Ribbon diagram of the crystal structure of AP2M1-pS616-IRS1 (PDB ID: 6BNT). b Surface drawing of AP2M1 colored by its electrostatic potential (blue, positive; red, negative; and white, neutral) with pS616-IRS1 shown as sticks.

juxtamembrane domain (Fig. 1a, b). The phosphotyrosine-binding domain of IRS proteins directly binds to the phosphorylated NPEY ${ }^{960}$ motif in $\mathrm{IR}^{80-84}$. The NPEY ${ }^{960}$ motif in IR had been implicated in AP2 binding and in IR endocytosis, but the mechanism remained unclear ${ }^{85,86}$. Activated IR phosphorylates several tyrosine residues in the IRS proteins, including multiple YXXФ motifs in the middle region (Fig. 1b) ${ }^{87,88}$. These phosphotyrosine motifs interact with PI3K, facilitating the activation of the PI3K-PKB/AKT pathway. SHP2 binds directly to the C-terminal phosphotyrosine residues in IRS proteins and dephosphorylates the tyrosine residues in the YXXФ motifs, thus negatively regulating PI3K activity ${ }^{89,90}$ (Fig. 1b, c). Multiple serine and threonine residues in IRS proteins can also be phosphorylated upon insulin stimulation ${ }^{91}$. The increased serine/threonine phosphorylation of IRS proteins is associated with insulin resistance in human and mouse models. ERK is one of the most well-known kinases of IRS proteins. Strikingly, the serine residues that follow the YXXФ motifs are phosphorylated by ERK ${ }^{50,92}$. ERKmediated phosphorylation of IRS proteins has been shown to reduce their tyrosine phosphorylation through negative feedback $^{50,91,92}$.

We have recently shown that IRS1 and IRS2 bind directly to the clathrin adaptor AP2M1 through multiple YXXФ motifs and promote insulin-activated IR endocytosis $^{21}$ (Fig. 1b). Interestingly, these AP2M1-YXXФ interactions are regulated by a phosphorylation switch mediated by ERK and SHP2. ERK-mediated serine/ threonine phosphorylation promotes SHP2-mediated tyrosine dephosphorylation of the YXXФ motifs, resulting in a switch from phosphotyrosine to phospho-serine/ threonine. Only phospho-serine/threonine-containing IRS proteins can interact with $\mathrm{AP} 2$ and trigger IR endocytosis.
The crystal structure of AP2M1 bound to serinephosphorylated $\mathrm{Y}^{612} \mathrm{XX} \Phi S^{616}$ motif in IRS1 (pS-IRS1) explains the structural basis of this phospho-switch (Fig. 2). Tyrosine (Y612) and methionine (M615) establish extensive hydrophobic interactions with AP2M1. The hydroxyl group at Y612 forms a hydrogen bond with D176 in AP2M1 (Fig. 2a). YXXФ IRS1 mutants in which tyrosine was replaced with alanine or phenylalanine showed weakened interaction with AP2M1 in vitro and IR endocytosis was not restored in IRS1-depleted cells. Tyrosine phosphorylation of the YXXФ motifs is expected to disrupt the IRS-AP2M1 interaction by introducing both static hindrance and unfavorable electrostatic interactions. In IRS1, pS616 is in the vicinity of a positively charged surface on the AP2M1, suggesting that this phospho-serine might participate in favorable electrostatic interactions with this basic region (Fig. 2b).

Consistent with the pS-IRS1-AP2M1 structure, pSIRS1 binds to AP2M1 with higher affinity, as compared to unphosphorylated IRS $1^{21}$. In addition, pS-IRS1 enhances the tyrosine dephosphorylation of IRS1 by SHP2. These data suggest that ERK-mediated serine phosphorylation of IRS proteins fosters AP2 interaction by directly enhancing the IRS-AP2M1 interaction and indirectly facilitating the SHP2-mediated tyrosine dephosphorylation of IRS, thus promoting clathrin-mediated IR endocytosis. Pharmacological SHP2 inhibition indeed blocks the insulinstimulated IRS1-AP2 interaction in hepatocytes and delays IR endocytosis. Taken together, these findings establish a direct function of the MAPK pathway in IR endocytosis and, possibly, in metabolic regulation.

In summary, there are two regulatory modules of IR endocytosis: the IRS module and the mitotic checkpoint module (Fig. 1b). The IRS module is activated by the SHP2-MAPK pathway, which is, in turn, activated by insulin signaling. These two modules collaboratively 
Table 1 Ongoing clinical trials and preclinical studies of SHP2 inhibitors.

\begin{tabular}{|c|c|c|c|}
\hline Inhibitor & Cancer type(s) & Clinical status & Notes $^{a}$ \\
\hline $\begin{array}{l}\text { JAB-3068 } \\
\text { (Jacobio Pharmaceuticals) }\end{array}$ & $\begin{array}{l}\text { Non-small cell lung cancer (NSCLC) } \\
\text { Head and neck cancer } \\
\text { Esophageal cancer }\end{array}$ & Phase $1 / 2 a$ & NCT03565003 \\
\hline $\begin{array}{l}\text { JAB-3312 } \\
\text { (Jacobio Pharmaceuticals) }\end{array}$ & $\begin{array}{l}\text { Non-small cell lung cancer (NSCLC) } \\
\text { Colorectal cancer } \\
\text { Pancreatic ductal carcinoma } \\
\text { Esophageal squamous cell carcinoma } \\
\text { Head and neck squamous cell } \\
\text { carcinoma } \\
\text { Breast cancer } \\
\text { Other solid tumors }\end{array}$ & Phase 1 & NCT04045496 \\
\hline $\begin{array}{l}\text { TN0155 } \\
\text { (Novartis) }\end{array}$ & $\begin{array}{l}\text { Non-small cell lung cancer (NSCLC) } \\
\text { Esophageal squamous cell cancer (SCC) } \\
\text { Head and neck SCC } \\
\text { Gastrointestinal stromal tumors }\end{array}$ & Phase $1 / 1 b$ & $\begin{array}{l}\text { NCT04000529 } \\
\text { NCT03114319 }\end{array}$ \\
\hline $\begin{array}{l}\text { RMC-4630 } \\
\text { (Revolution Medicines) }\end{array}$ & Solid tumors (unspecified) & Phase $1 \mathrm{~b} / 2$ & NCT03989115 \\
\hline $\begin{array}{l}\text { RLY-1971 } \\
\text { (Relay Therapeutics) }\end{array}$ & Solid tumors (unspecified) & Phase 1 & NCT04252339 \\
\hline SHP099 & $\begin{array}{l}\text { Esophageal cancer cells } \\
\text { Hematopoietic cancer cells } \\
\text { Colorectal cancer cells } \\
\text { KRAS-mutant cancer cells } \\
\text { Triple-negative breast cancer }\end{array}$ & $\begin{array}{l}\text { No clinical trials; research involves cell lines and mouse } \\
\text { xenografts } 57,100,106 \text {. }\end{array}$ & \\
\hline
\end{tabular}

${ }^{a}$ https://clinicaltrials.gov/ct2/ (identification number).

${ }^{\mathrm{b}}$ Combination with spartalizumab or ribociclib.

promote the selective endocytosis of activated IR. Our studies suggest that targeting the feedback regulation of IR endocytosis might be beneficial for diabetes treatment. As SHP2 promotes IR endocytosis directly by removing IRS tyrosine phosphorylation and also indirectly by activating the MAPK pathway, inhibition of SHP2 is expected to disrupt the feedback loop, prolong insulin signaling at the PM, and improve insulin sensitivity.

\section{The use of SHP2 inhibitors for the treatment of cancer and} diabetes

Because of its role in growth factor receptor signaling, SHP2 has been implicated in the development of many diseases. Mutations of SHP2 are associated with multiple disorders, most notably Noonan syndrome and LEOPARD syndrome ${ }^{93}$. Somatic SHP2 mutations are also associated with cases of childhood leukemia, including juvenile myelomonocytic leukemia, myelodysplastic syndrome, and acute myeloid leukemia ${ }^{94,95}$. SHP2 overexpression is also causally related to cell proliferation dysfunction. Suppressing SHP2 expression in adult leukemia increases apoptosis and reduces the growth of leukemia cells ${ }^{96}$. Similarly, SHP2 levels are elevated in other types of cancer, including breast and ovarian cancers $^{97,98}$. Due to the association of SHP2 with cancer cell proliferation, SHP2 has emerged as a potential target for cancer therapy.

SHP2 undergoes autoinhibition in the absence of an activator; when not stimulated by insulin, the N-SH2 domain binds the PTP domain and blocks its active site (Fig. 1c) ${ }^{99}$. The small-molecule SHP2 allosteric inhibitor SHP099 takes advantage of this natural regulatory mechanism by interacting with all three domains of SHP2 when it is in the autoinhibited configuration, locking it into the inactive form ${ }^{57}$. SHP099 binds to SHP2 with a high degree of specificity. Strikingly, SHP099 shows no inhibitory activity against SHP1, the closest homolog of SHP2. The effectiveness of SHP099 as an allosteric SHP2 inhibitor makes it a viable option for proof-of-principle studies on SHP2-induced inhibition as a cancer therapy.

Several clinical studies are currently exploring the use of SHP2 inhibitors to treat RTK-mutated cancers (Table 1). SHP099 has been used in conjunction with MEK inhibitors to decrease cancer cell proliferation in multiple types 


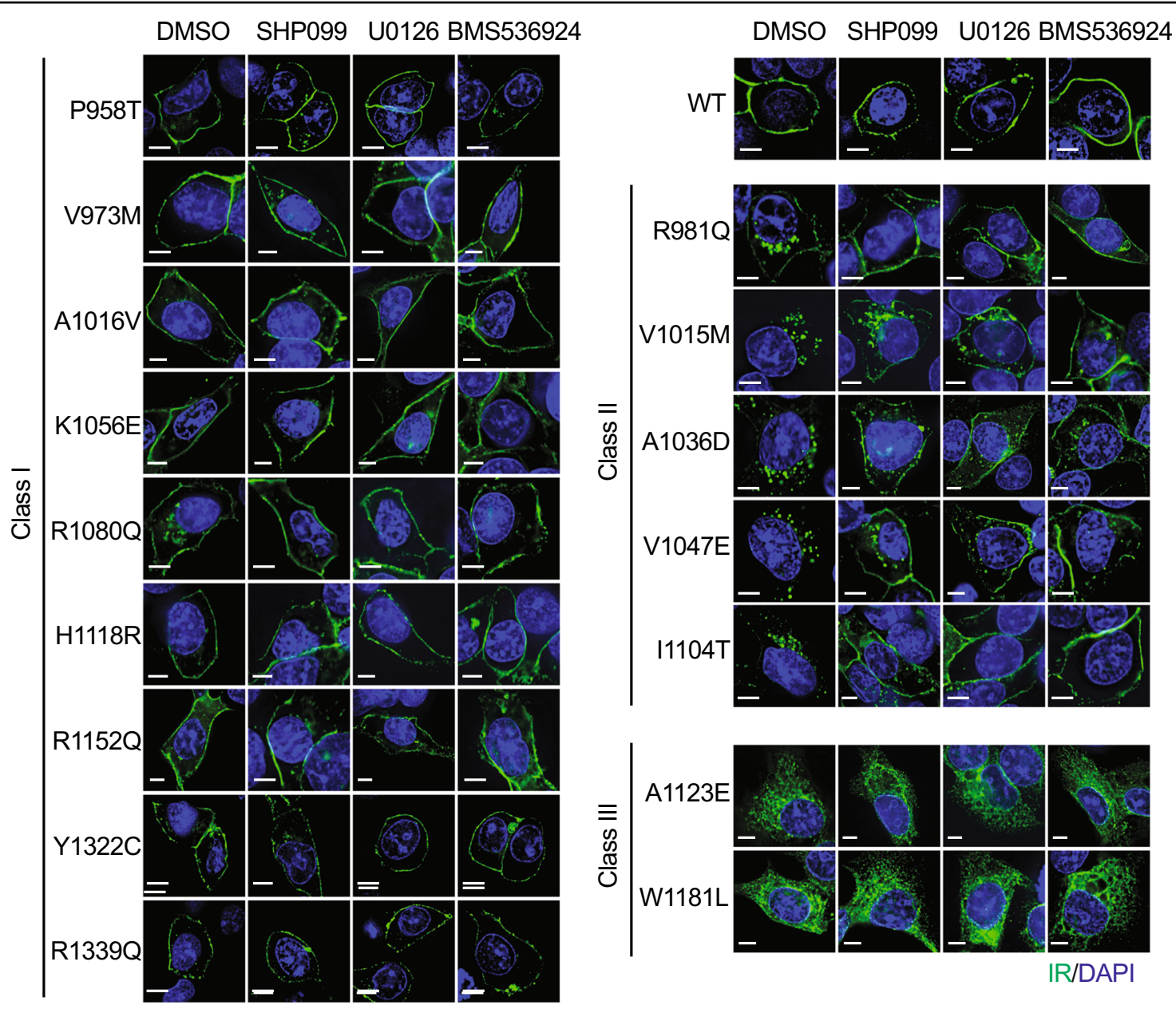

Fig. 3 Characterization of IR mutations found in human patients. HepG2 cells expressing IR-GFP wild-type (WT) or mutants were starved for $14 \mathrm{~h}$, treated with the indicated inhibitors for $4 \mathrm{~h}$, and stained with anti-GFP (IR; green; 1181446001, Sigma) and DAPI (blue). SHP099 (SHP2 inhibitor, 10 MM, MedChemExpress), U0126 (MEK inhibitor, $40 \mu$ M, Cell Signaling), and BMS536924 (IR kinase inhibitor, $2 \mu$ M, Tocris). Scale bar, $5 \mu \mathrm{m}$.

of cancer. This method successfully mitigated the development of the adaptive resistance to MEK inhibition that occurs when MEK inhibitors are used alone ${ }^{100}$. SHP099 is effective in decreasing tumor burden and promoting antitumor immunity in mice with grafted colon cancer cells $^{101}$. These findings suggest that SHP099 is a promising candidate for cancer therapy, both as a monotherapy and in conjunction with other agents.

Studies on SHP2 and its associated signaling pathways have also revealed its involvement in the regulation of insulin signaling. Patients with LEOPARD syndromerelated SHP2 mutations exhibit resistance to diet-induced obesity, an improved overall metabolic profile, and insulin hypersensitivity ${ }^{102}$. This outcome suggests a possible role for SHP2 inhibition or modulation in treating insulin resistance. Our study has shown that SHP2 inhibitors can be potentially repurposed to treat type 2 diabetes ${ }^{21}$. These results follow the trend shown in previous research indicating a relationship between SHP2 and insulin signaling and suggest SHP2 inhibition as a promising therapeutic method for not only cancer treatment, but also treatment of insulin resistance and diabetes. Cancer and diabetes share common risk factors such as obesity, hyperinsulinemia, and aging. The number of patients suffering from both diseases has increased dramatically in recent years. SHP2 inhibitors may be particularly beneficial to patients who have both diabetes and cancer.

\section{Targeting IR endocytosis for insulin resistance treatment}

Mutations of IR are known to cause inherited severe insulin resistance syndromes ${ }^{103}$, but the mechanisms by which these mutations affect IR function have not been systematically explored. Using the missense mutations in the cytoplasmic region of IR found in patients with severe insulin resistance ${ }^{103}$, we defined three distinct classes of IR mutants based on their subcellular localization in the unstimulated state (Fig. 3 and Table 2). Class I mutants localize to the PM. Class II mutants show reduced signals 
Table 2 Characterization of IR mutations found in human patients.

\begin{tabular}{|c|c|c|c|c|c|}
\hline Class & $\begin{array}{l}\text { Mutation HGVS } \\
\text { nomenclature }\end{array}$ & $\begin{array}{l}\text { Mutation mature, long } \\
\text { isoform }\end{array}$ & $\begin{array}{l}\text { Mutation mature, short } \\
\text { isoform }\end{array}$ & Localization $^{\mathrm{b}}$ & Phenotype \\
\hline \multirow[t]{9}{*}{ | } & P997T & Р970T & P958T & PM & $\begin{array}{l}\text { Rabson-Mendenhall } \\
\text { syndrome }\end{array}$ \\
\hline & V1012M & V985M & V973M & PM & Type 2 diabetes \\
\hline & A1055V & A1028V & A1016V & PM & Insulin resistance \\
\hline & K1095E & K1068E & K1056E & PM & Type 2 diabetes \\
\hline & R1119Q & R1092Q & R1080Q & PM & Leprechaunism \\
\hline & H1157R & H1130R & H1118R & PM & Insulin resistance \\
\hline & R1191Q & R1164Q & R1152Q & PM & Type 2 diabetes \\
\hline & Y1361C & Y1334C & Y1322C & PM & Type 2 diabetes \\
\hline & R1378Q & R1351Q & R1339Q & PM & Insulin resistance \\
\hline \multirow[t]{5}{*}{$\|$} & R1020Q & R993Q & R981Q & IC & Insulin resistance \\
\hline & V1054M & V1027M & V1015M & IC & Leprechaunism \\
\hline & A1075D & A1048D & A1036D & IC & Insulin resistance \\
\hline & V1086E & V1059E & V1047E & IC & Type 2 diabetes \\
\hline & I1143T & I1116T & I1104T & IC & $\begin{array}{l}\text { Rabson-Mendenhall } \\
\text { syndrome }\end{array}$ \\
\hline \multirow[t]{2}{*}{ III } & A1162E & A1135E & A1123E & ER/Golgi & Insulin resistance \\
\hline & W1220L & W1193L & A1181L & ER/Golgi & Insulin resistance \\
\hline
\end{tabular}

$E R /$ Golgi endoplasmic reticulum/Golgi apparatus, IC intracellular compartment, $P M$ plasma membrane.

${ }^{a}$ Human Genome Variation Society, http://www.hgvs.org/rec.html.

${ }^{\mathrm{b}}$ The cellular localization of IR-GFP in the basal, unstimulated state.

at the PM and are enriched in RAB7-positive intracellular compartments (Fig. 4). Class III mutants remain in the ER and the Golgi apparatus, indicating that class III mutations affect IR processing and trafficking. The addition of dynasore, a chemical inhibitor of dynamin, elevated the IR level of the class II mutants at the PM (Fig. 4), suggesting that class II mutations cause premature CME of IR prior to insulin stimulation.

Treating cells with SHP099 and U0126, a MEK inhibitor, significantly enhanced the PM levels of class II IR mutants, but not those of class I and III IR mutants. These results suggest that targeting IR endocytosis can potentially alleviate insulin resistance in patients with class II IR mutations and possibly in other type 2 diabetes patients. Future studies are required to determine the role of premature IR endocytosis in the pathogenesis of human insulin resistance. Our current study monitored IR endocytosis in HepG2 cells that expressed endogenous IR. Only patients with both alleles of IR mutated displayed insulin resistance phenotypes, whereas their parents, who each had a single mutated allele, did not exhibit these phenotypes. To mimic the situation of the patients, we depleted endogenous IR in HepG2 cells; however, due to high levels of cell death, we could not assess whether the premature endocytosis of IR mutants occurred in the absence of endogenous wild-type IR. It will be interesting to examine the IR PM levels before and after insulin stimulation in patient cell lines that harbor the particular class II mutations and to determine whether inhibitors of SHP2 or the MAPK pathway can recover the IR PM levels and insulin sensitivity.

\section{Perspective}

Our recent studies provide further mechanistic insight into IR endocytosis and raise many interesting questions as follows: (1) the core components of MCC, including MAD2, BUBR1, and CDC20, are assembled onto IR to control its endocytosis in interphase. Is the mitotic module regulated by the SHP2-MAPK pathway during insulin signaling? If it is, what is the main target of SHP2 and MAPK in this module? (2) The fact that an MCC-like complex is assembled onto IR suggests that IR might reciprocally control MCC assembly and spindle checkpoint signaling during cell division. Can insulin and the metabolic environment control genomic stability through IR? (3) How do the two modules-the IRS and mitotic checkpoint modules-cooperate to promote IR endocytosis? How many copies of the AP2 complex, BUBR1, or 


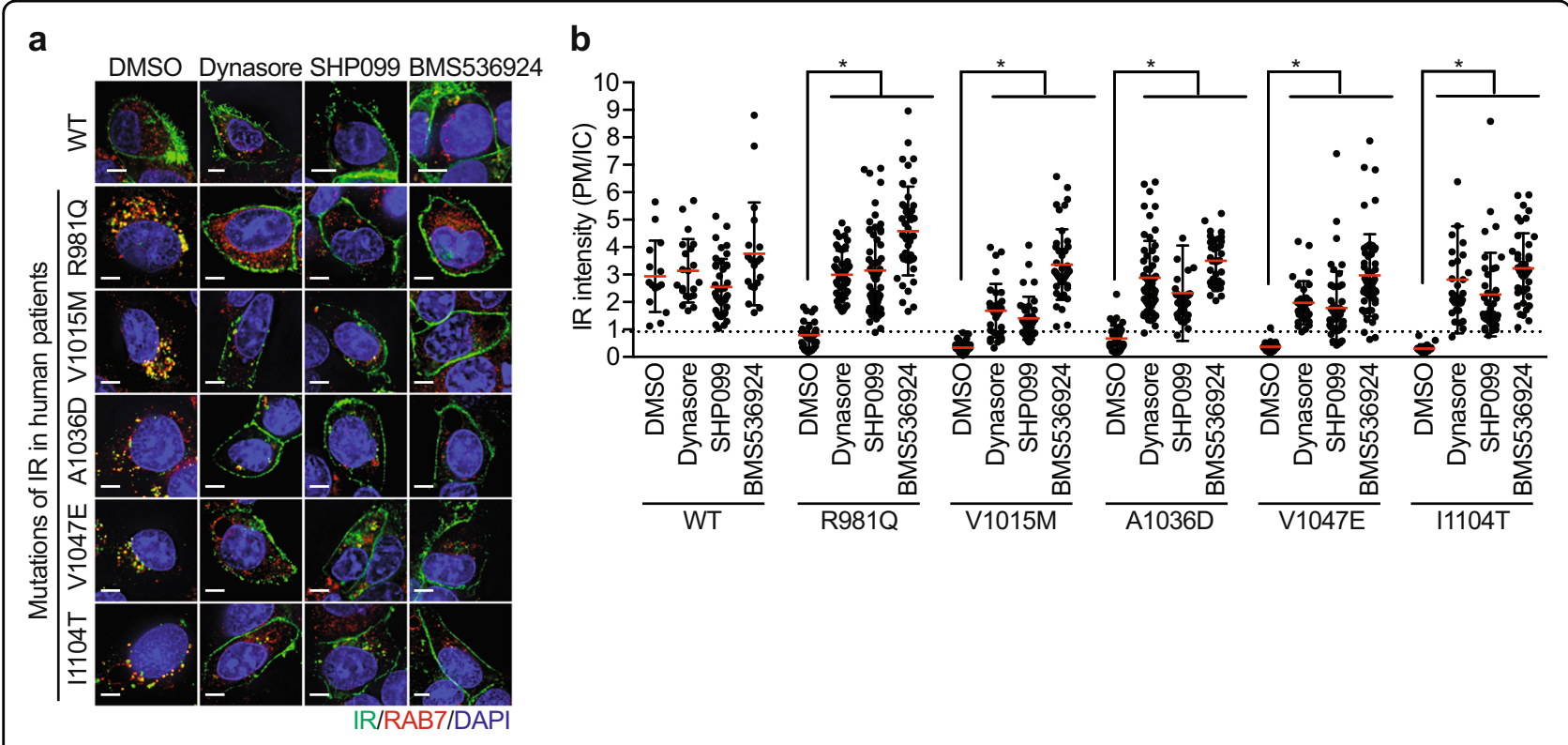

Fig. 4 Characterization of class II IR mutations found in human patients. a HepG2 cells stably expressing IR-GFP WT or Class II mutants were serum starved for $14 \mathrm{~h}$, treated with the indicated inhibitors for $4 \mathrm{~h}$, and stained with anti-GFP (IR; green) and anti-RAB7 (Red; D95F2, Cell Signaling) antibodies. Dynasore (dynamin inhibitor, $80 \mu \mathrm{M}$, Sigma), SHP099 (SHP2 inhibitor, $10 \mu \mathrm{M}$, MedChemExpress), and BMS536924 (IR kinase inhibitor, $2 \mu$ M, Tocris). Scale bar, $5 \mu \mathrm{m}$. b Quantification of the ratios of PM and IC IR-GFP signals of the cells shown in $\mathbf{a}$ (mean \pm SD; ${ }^{*} p<0.0001$ ).

IRS1/2 are recruited to each IR dimer? Do two modules engage a single AP2 complex? This is theoretically possible because BUBR1 and IRS1/2 do not bind the same site on AP2: BUBR1 binds to AP2B1, and IRS1/2 bind to AP2M1. (4) What is the physiological function of the feedback regulation on human insulin resistance? Can hyperactivation of the MAPK pathway by altered metabolic stress reduce the IR levels at the PM? (5) Are the IR PM levels reduced in human patients harboring class II mutations? Can inhibitors of SHP2 or the MAPK pathway recover the IR PM levels in these patients and improve insulin sensitivity? Future studies aimed at answering these questions will provide further insight into the pathogenesis of insulin resistance.

Type 1 insulin-like growth factor receptor (IGF1R) belongs to the IR family. The intracellular domains of IR and IGF1R share over $80 \%$ of amino acid identity. Although IR and IGF1R also share several common adaptors and effectors for downstream signaling pathways, IGF1R only controls cell growth and proliferation, whereas IR controls both cell growth and metabolic homeostasis. The mechanisms by which these two highly homologous receptors achieve different signaling outcomes are largely unknown. In the context of endocytosis, IGF1R binds to IRS1-AP2 but does not have MIM ${ }^{16,40}$, suggesting that the internalization of IGF1R does not involve the mitotic checkpoint module and is solely controlled by the IRS module. Strikingly, Yoneyama et al. ${ }^{104}$ showed that IRS1, but not IRS2, negatively regulates IGF1R endocytosis. Most importantly, the timing of endocytosis after the activation of IGF1R is very different from that of IR. In contrast to activated IR, which is internalized within minutes, activated IGF1R can remain at the PM for over $1 \mathrm{~h}$. Recent cryo-EM structural studies showed that the $\Gamma$-shaped asymmetric IGF1R dimer was bound to only one IGF1 molecule, while the T-shaped symmetric IR dimer was bound to multiple insulin molecules ${ }^{45,105}$. Future studies are required to explore whether these important structural differences upon ligand binding affect the endocytosis and downstream signaling of IR and IGF1R.

\section{Author details \\ ${ }^{1}$ Department of Pathology and Cell Biology, Vagelos College of Physicians and Surgeons, Columbia University, 630 West 168th Street, New York, NY 10032, USA. 'Laboratory of Cell Biology, School of Life Sciences, Westlake University, Hangzhou, Zhejiang 310024, China. ${ }^{3}$ Department of Pharmacology, University of Texas Southwestern Medical Center, 6001 Forest Park Road, Dallas, TX 75390, USA}

Conflict of interest

The authors declare that they have no conflict of interest.

Publisher's note

Springer Nature remains neutral with regard to jurisdictional claims in published maps and institutional affiliations.

Received: 30 March 2020 Accepted: 11 May 2020.

Published online: 23 June 2020 


\section{References}

1. Petersen, M. C. \& Shulman, G. I. Mechanisms of insulin action and insulin resistance. Physiol. Rev. 98, 2133-2223 (2018).

2. Haeusler, R. A., McGraw, T. E. \& Accili, D. Biochemical and cellular properties of insulin receptor signalling. Nat. Rev. Mol. Cell Biol. 19, 31-44 (2018).

3. Reaven, G. M. Banting lecture 1988. Role of insulin resistance in human disease. Diabetes 37, 1595-1607 (1988).

4. Facchini, F. S., Hua, N., Abbasi, F. \& Reaven, G. M. Insulin resistance as a predictor of age-related diseases. J. Clin. Endocrinol. Metab. 86, 3574-3578 (2001).

5. Lillioja, S. et al. Insulin resistance and insulin secretory dysfunction as precursors of non-insulin-dependent diabetes mellitus. Prospective studies of Pima Indians. N. Engl. J. Med 329, 1988-1992 (1993).

6. Warram, J. H., Martin, B. C., Krolewski, A. S., Soeldner, J. S. \& Kahn, C. R. Slow glucose removal rate and hyperinsulinemia precede the development of type II diabetes in the offspring of diabetic parents. Ann. Intern Med 113, 909-915 (1990).

7. Kahn, S. E. The relative contributions of insulin resistance and beta-cell dysfunction to the pathophysiology of type 2 diabetes. Diabetologia $\mathbf{4 6}$, 3-19 (2003).

8. Kasuga, M. Insulin resistance and pancreatic beta cell failure. J. Clin. Invest 116 1756-1760 (2006).

9. Schwartz, S. S. et al. The time is right for a new classification system for diabetes: rationale and implications of the beta-cell-centric classification schema. Diabetes Care 39, 179-186, https://doi.org/10.2337/dc15-1585 (2016).

10. Schwartz, S. S. et al. A unified pathophysiological construct of diabetes and its complications. Trends Endocrinol. Metab. 28, 645-655 (2017).

11. Shanik, M. H. et al. Insulin resistance and hyperinsulinemia: is hyperinsulinemia the cart or the horse? Diabetes Care 31(Suppl 2), S262-268 (2008).

12. Gregory, J. M. et al. latrogenic hyperinsulinemia, not hyperglycemia, drives insulin resistance in type 1 diabetes as revealed by comparison with GCKMODY (MODY2). Diabetes 68, 1565-1576 (2019).

13. Knutson, V. P., Donnelly, P. V., Balba, Y. \& Lopez-Reyes, M. Insulin resistance is mediated by a proteolytic fragment of the insulin receptor. J. Biol. Chem. $\mathbf{2 7 0}$ 24972-24981 (1995).

14. Czech, M. P. Insulin action and resistance in obesity and type 2 diabetes. Nat. Med 23, 804-814 (2017).

15. Goh, L. K. \& Sorkin, A. Endocytosis of receptor tyrosine kinases. Cold Spring Harb. Perspect. Biol. 5, a017459 (2013).

16. Choi, E. \& Yu, H. Spindle checkpoint regulators in insulin signaling. Front Cell Dev. Biol. 6, 161 (2018).

17. Traub, L. M. \& Bonifacino, J. S. Cargo recognition in clathrin-mediated endocytosis. Cold Spring Harb. Perspect. Biol. 5, a016790 (2013).

18. Kaksonen, M. \& Roux, A. Mechanisms of clathrin-mediated endocytosis. Nat. Rev. Mol. Cell Biol. 19, 313-326 (2018).

19. Soll, A. H., Kahn, C. R. \& Neville, D. M. Jr. Insulin binding to liver plasm membranes in the obese hyperglycemic (ob/ob) mouse. Demonstration of a decreased number of functionally normal receptors. J. Biol. Chem. 250 4702-4707 (1975).

20. Caro, J. F. et al. Studies on the mechanism of insulin resistance in the liver from humans with noninsulin-dependent diabetes. Insulin action and binding in isolated hepatocytes, insulin receptor structure, and kinase activity. J. Clin. Invest 78, 249-258 (1986)

21. Choi, E. et al. Mitotic regulators and the SHP2-MAPK pathway promote IR endocytosis and feedback regulation of insulin signaling. Nat. Commun. 10 1473, https://doi.org/10.1038/s41467-019-09318-3 (2019).

22. Musacchio, A. The molecular biology of spindle assembly checkpoint signaling dynamics. Curr. Biol. 25, R1002-1018 (2015).

23. Foley, E. A. \& Kapoor, T. M. Microtubule attachment and spindle assembly checkpoint signalling at the kinetochore. Nat. Rev. Mol. Cell Biol. 14, 25-37 (2013).

24. Jia, L., Kim, S. \& Yu, H. Tracking spindle checkpoint signals from kinetochores to APC/C. Trends Biochem Sci. 38, 302-311 (2013).

25. London, N. \& Biggins, S. Signalling dynamics in the spindle checkpoint response. Nat. Rev. Mol. Cell Biol. 15, 736-747 (2014).

26. Holland, A. J. \& Cleveland, D. W. Boveri revisited: chromosomal instability, aneuploidy and tumorigenesis. Nat. Rev. Mol. Cell Biol. 10, 478-487 (2009).

27. Pfau, S. J. \& Amon, A. Chromosomal instability and aneuploidy in cancer: from yeast to man. EMBO Rep. 13, 515-527 (2012).

28. Peters, J. M. The anaphase promoting complex/cyclosome: a machine designed to destroy. Nat. Rev. Mol. Cell Biol. 7, 644-656 (2006).
29. Yu, H. Cdc20: a WD40 activator for a cell cycle degradation machine. Mol. Cell 27, 3-16 (2007).

30. Luo, X. \& Yu, H. Protein metamorphosis: the two-state behavior of Mad2. Structure 16, 1616-1625 (2008).

31. Izawa, D. \& Pines, J. The mitotic checkpoint complex binds a second CDC20 to inhibit active APC/C. Nature 517, 631-634 (2015).

32. Sudakin, V., Chan, G. K. \& Yen, T. J. Checkpoint inhibition of the APC/C in HeLa cells is mediated by a complex of BUBR1, BUB3, CDC20, and MAD2. J. Cell Biol. 154, 925-936 (2001).

33. Mapelli, M. \& Musacchio, A. MAD contortions: conformational dimerization boosts spindle checkpoint signaling. Curr. Opin. Struct. Biol. 17, 716-725 (2007)

34. Choi, E. et al. BubR1 acetylation at prometaphase is required for modulating APC/C activity and timing of mitosis. EMBO J. 28, 2077-2089 (2009).

35. Hagan, R. S. et al. p31(comet) acts to ensure timely spindle checkpoint silencing subsequent to kinetochore attachment. Mol. Biol. Cell 22, 4236-4246 (2011)

36. Jia, L. et al. Defining pathways of spindle checkpoint silencing: functional redundancy between Cdc20 ubiquitination and p31(comet). Mol. Biol. Cell 22, 4227-4235, https:/doi.org/10.1091/mbc.E11-05-0389 (2011).

37. Xia, G. et al. Conformation-specific binding of p31(comet) antagonizes the function of Mad2 in the spindle checkpoint. EMBO J. 23, 3133-3143 (2004).

38. Yang, M. et al. p31comet blocks Mad2 activation through structural mimicry. Cell 131, 744-755, https://doi.org/10.1016/j.cell.2007.08.048 (2007).

39. Habu, T., Kim, S. H., Weinstein, J. \& Matsumoto, T. Identification of a MAD2binding protein, CMT2, and its role in mitosis. EMBO J. 21, 6419-6428 (2002).

40. Choi, E., Zhang, X., Xing, C. \& Yu, H. Mitotic checkpoint regulators control insulin signaling and metabolic homeostasis. Cell 166, 567-581 (2016).

41. Baker, D. J. et al. Opposing roles for p16lnk4a and p19Arf in senescence and ageing caused by BubR1 insufficiency. Nat. Cell Biol. 10, 825-836 (2008).

42. Grako, K. A., Olefsky, J. M. \& McClain, D. A. Tyrosine kinase-defective insulin receptors undergo decreased endocytosis but do not affect internalization of normal endogenous insulin receptors. Endocrinology 130, 3441-3452 (1992)

43. Carpentier, J. L. et al. Two steps of insulin receptor internalization depend on different domains of the beta-subunit. J. Cell Biol. 122, 1243-1252 (1993).

44. De Meyts, P. The Insulin Receptor and Its Signal Transduction Network. In: Endotext (eds Feingold, K. R., Anawalt, B. \& Boyce, A. et al.) (MDText.com, Inc: South Dartmouth (MA), 2000).

45. Uchikawa, E., Choi, E., Shang, G., Yu, H. \& Bai, X. C. Activation mechanism of the insulin receptor revealed by cryo-EM structure of the fully liganded receptor-ligand complex. Elife 8, https://doi.org/10.7554/eLife.48630 (2019).

46. Scapin, G. et al. Structure of the insulin receptor-insulin complex by singleparticle cryo-EM analysis. Nature 556, 122-125 (2018).

47. Weis, F. et al. The signalling conformation of the insulin receptor ectodomain. Nat. Commun. 9, 4420 (2018)

48. Boucher, J., Kleinridders, A. \& Kahn, C. R. Insulin receptor signaling in normal and insulin-resistant states. Cold Spring Harb Perspect Biol 6, a009191 https:// doi.org/10.1101/cshperspect.a009191 (2014).

49. White, M. F. Insulin signaling in health and disease. Science 302, 1710-1711 (2003).

50. De Fea, K. \& Roth, R. A. Modulation of insulin receptor substrate-1 tyrosine phosphorylation and function by mitogen-activated protein kinase. J. Biol. Chem. 272, 31400-31406 (1997)

51. Zheng, Y. et al. Improved insulin sensitivity by calorie restriction is associated with reduction of ERK and p70S6K activities in the liver of obese Zucker rats. J. Endocrinol. 203, 337-347 (2009).

52. Banks, A. S. et al. An ERK/Cdk5 axis controls the diabetogenic actions of PPARgamma. Nature 517, 391-395 (2015).

53. Gehart, H., Kumpf, S., Ittner, A. \& Ricci, R. MAPK signalling in cellular metabolism: stress or wellness? EMBO Rep. 11, 834-840 (2010)

54. Pronk, G. J., McGlade, J., Pelicci, G., Pawson, T. \& Bos, J. L. Insulin-induced phosphorylation of the 46- and 52-kDa Shc proteins. J. Biol. Chem. 268 5748-5753 (1993).

55. Neel, B. G., Gu, H. \& Pao, L. The 'Shp'ing news: SH2 domain-containing tyrosine phosphatases in cell signaling. Trends Biochem Sci. 28, 284-293 (2003)

56. Furcht, C. M., Buonato, J. M. \& Lazzara, M. J. EGFR-activated Src family kinases maintain GAB1-SHP2 complexes distal from EGFR. Sci. Signal 8, ra46 (2015).

57. Chen, Y. N. et al. Allosteric inhibition of SHP2 phosphatase inhibits cancers driven by receptor tyrosine kinases. Nature 535, 148-152 (2016). 
58. Nichols, R. J. et al. RAS nucleotide cycling underlies the SHP2 phosphatase dependence of mutant BRAF-, NF1- and RAS-driven cancers. Nat. Cell Biol. 20 1064-1073 (2018).

59. Cobb, M. H. MAP kinase pathways. Prog. Biophys. Mol. Biol. 71, 479-500 (1999).

60. Roth, G. et al. MAP kinases Erk1/2 phosphorylate sterol regulatory elementbinding protein (SREBP)-1a at serine 117 in vitro. J. Biol. Chem. 275, 33302-33307 (2000).

61. Kotzka, J. et al. Insulin-activated Erk-mitogen-activated protein kinases phosphorylate sterol regulatory element-binding Protein-2 at serine residues 432 and 455 in vivo. J. Biol. Chem. 279, 22404-22411 (2004).

62. Yang, L., Zheng, L., Chng, W. J. \& Ding, J. L. Comprehensive analysis of ERK1/ 2 substrates for potential combination immunotherapies. Trends Pharm. Sci. 40, 897-910 (2019)

63. Unal, E. B., Uhlitz, F. \& Bluthgen, N. A compendium of ERK targets. FEBS Lett. 591, 2607-2615 (2017).

64. Wortzel, I. \& Seger, R. The ERK cascade: distinct functions within various subcellular organelles. Genes Cancer 2, 195-209 (2011).

65. Hatano, N. et al. Essential role for ERK2 mitogen-activated protein kinase in placental development. Genes Cells 8, 847-856 (2003).

66. Saba-El-Leil, M. K. et al. An essential function of the mitogen-activated protein kinase Erk2 in mouse trophoblast development. EMBO Rep. 4, 964-968 (2003).

67. Yao, Y. et al. Extracellular signal-regulated kinase 2 is necessary for mesoderm differentiation. Proc. Natl Acad. Sci. USA 100, 12759-12764 (2003).

68. Pages, G. et al. Defective thymocyte maturation in p44 MAP kinase (Erk 1) knockout mice. Science 286, 1374-1377 (1999).

69. Jager, J. et al. Deficiency in the extracellular signal-regulated kinase 1 (ERK1) protects leptin-deficient mice from insulin resistance without affecting obesity. Diabetologia 54, 180-189 (2011)

70. Bost, F. et al. The extracellular signal-regulated kinase isoform ERK1 is specifically required for in vitro and in vivo adipogenesis. Diabetes 54, 402-411 (2005).

71. Bouzakri, K. et al. Reduced activation of phosphatidylinositol-3 kinase and increased serine 636 phosphorylation of insulin receptor substrate-1 in primary culture of skeletal muscle cells from patients with type 2 diabetes. Diabetes 52, 1319-1325 (2003).

72. Carlson, C. J., Koterski, S., Sciotti, R. J., Poccard, G. B. \& Rondinone, C. M. Enhanced basal activation of mitogen-activated protein kinases in adipocytes from type 2 diabetes: potential role of p38 in the downregulation of GLUT4 expression. Diabetes 52, 634-641 (2003).

73. Ozaki, K. I. et al. Targeting the ERK signaling pathway as a potential treatment for insulin resistance and type 2 diabetes. Am. J. Physiol. Endocrinol. Metab. 310, E643-E651 (2016).

74. Saxton, T. M. et al. Abnormal mesoderm patterning in mouse embryos mutant for the SH2 tyrosine phosphatase Shp-2. EMBO J. 16, 2352-2364 (1997).

75. Princen, F. et al. Deletion of Shp2 tyrosine phosphatase in muscle leads to dilated cardiomyopathy, insulin resistance, and premature death. Mol. Cell Biol. 29, 378-388 (2009)

76. Zhang, E. E., Chapeau, E., Hagihara, K. \& Feng, G. S. Neuronal Shp2 tyrosine phosphatase controls energy balance and metabolism. Proc. Natl Acad. Sci. USA 101, 16064-16069 (2004).

77. Matsuo, $\mathrm{K}$ et al. Altered glucose homeostasis in mice with liver-specific deletion of Src homology phosphatase 2. J. Biol. Chem. 285, 39750-39758 (2010).

78. Nagata, N. et al. Hepatic Src homology phosphatase 2 regulates energy balance in mice. Endocrinology 153, 3158-3169 (2012).

79. Bard-Chapeau, E. A. et al. Deletion of Gab1 in the liver leads to enhanced glucose tolerance and improved hepatic insulin action. Nat. Med 11, 567-571 (2005).

80. White, M. F. et al. Mutation of the insulin receptor at tyrosine 960 inhibits signal transmission but does not affect its tyrosine kinase activity. Cell $\mathbf{5 4}$ 641-649 (1988).

81. Eck, M. J., Dhe-Paganon, S., Trub, T., Nolte, R. T. \& Shoelson, S. E. Structure of the IRS-1 PTB domain bound to the juxtamembrane region of the insulin receptor. Cell 85, 695-705 (1996).

82. Gustafson, T. A., He, W., Craparo, A., Schaub, C. D. \& O'Neill, T. J. Phosphotyrosine-dependent interaction of SHC and insulin receptor substrate 1 with the NPEY motif of the insulin receptor via a novel non-SH2 domain. Mol. Cell Biol. 15, 2500-2508 (1995).
83. He, W. et al. Interaction of insulin receptor substrate-2 (IRS-2) with the insulin and insulin-like growth factor I receptors. Evidence for two distinct phosphotyrosine-dependent interaction domains within IRS-2. J. Biol. Chem. 271, 11641-11645 (1996).

84. Wolf, G. et al. PTB domains of IRS-1 and Shc have distinct but overlapping binding specificities. J. Biol. Chem. 270, 27407-27410 (1995).

85. Backer, J. M., Kahn, C. R., Cahill, D. A., Ullrich, A. \& White, M. F. Receptormediated internalization of insulin requires a 12-amino acid sequence in the juxtamembrane region of the insulin receptor beta-subunit. J. Biol. Chem 265, 16450-16454 (1990).

86. Backer, J. M., Shoelson, S. E., Haring, E. \& White, M. F. Insulin receptors internalize by a rapid, saturable pathway requiring receptor autophosphorylation and an intact juxtamembrane region. J. Cell Biol. 115 1535-1545 (1991).

87. Sun, X. J., Crimmins, D. L., Myers, M. G. Jr., Miralpeix, M. \& White, M. F. Pleiotropic insulin signals are engaged by multisite phosphorylation of IRS-1. Mol. Cell Biol. 13, 7418-7428 (1993).

88. White, M. F. The insulin signalling system and the IRS proteins. Diabetologia 40(Suppl 2), S2-17 (1997)

89. Myers, M. G. Jr. et al. The $\mathrm{COOH}$-terminal tyrosine phosphorylation sites on IRS-1 bind SHP-2 and negatively regulate insulin signaling. J. Biol. Chem. 273 26908-26914 (1998).

90. Zhang, S. Q. et al. Receptor-specific regulation of phosphatidylinositol 3'kinase activation by the protein tyrosine phosphatase Shp2. Mol. Cell Biol. 22, 4062-4072 (2002).

91. Copps, K. D. \& White, M. F. Regulation of insulin sensitivity by serine/threonine phosphorylation of insulin receptor substrate proteins IRS1 and IRS2. Diabetologia 55, 2565-2582 (2012).

92. Hers, I. \& Tavare, J. M. Mechanism of feedback regulation of insulin receptor substrate-1 phosphorylation in primary adipocytes. Biochem J. 388, 713-720 (2005).

93. Edouard, T. et al. Functional effects of PTPN11 (SHP2) mutations causing LEOPARD syndrome on epidermal growth factor-induced phosphoinositide 3-kinase/AKT/glycogen synthase kinase 3beta signaling. Mol. Cell Biol. 30 2498-2507 (2010).

94. Tartaglia, M. \& Gelb, B. D. Germ-line and somatic PTPN11 mutations in human disease. Eur. J. Med Genet 48, 81-96 (2005).

95. Tartaglia, M. et al. Somatic mutations in PTPN11 in juvenile myelomonocytic leukemia, myelodysplastic syndromes and acute myeloid leukemia. Nat. Genet 34, 148-150 (2003).

96. Xu, R. et al. Overexpression of Shp2 tyrosine phosphatase is implicated in leukemogenesis in adult human leukemia. Blood 106, 3142-3149 (2005).

97. Aceto, N. et al. Tyrosine phosphatase SHP2 promotes breast cancer progression and maintains tumor-initiating cells via activation of key transcription factors and a positive feedback signaling loop. Nat. Med 18, 529-537 (2012).

98. Hu, Z., Li, J., Gao, Q., Wei, S. \& Yang, B. SHP2 overexpression enhances the invasion and metastasis of ovarian cancer in vitro and in vivo. Onco Targets Ther. 10, 3881-3891 (2017).

99. Grossmann, K. S., Rosario, M., Birchmeier, C. \& Birchmeier, W. The tyrosine phosphatase Shp2 in development and cancer. Adv. Cancer Res 106, 53-89 (2010)

100. Fedele, C. et al. SHP2 inhibition prevents adaptive resistance to MEK inhibitors in multiple cancer models. Cancer Discov. 8, 1237-1249 (2018).

101. Zhao, M. et al. SHP2 inhibition triggers anti-tumor immunity and synergizes with PD-1 blockade. Acta Pharm. Sin. B 9, 304-315 (2019).

102. Tajan, M. et al. LEOPARD syndrome-associated SHP2 mutation confers leanness and protection from diet-induced obesity. Proc. Natl Acad. Sci. USA 111, E4494-4503 (2014).

103. Ardon, O., Procter, M., Tvrdik, T., Longo, N. \& Mao, R. Sequencing analysis of insulin receptor defects and detection of two novel mutations in INSR gene. Mol. Genet. Metab. Rep. 1, 71-84 (2014).

104. Yoneyama, Y. et al. IRS-1 acts as an endocytic regulator of IGF-I receptor to facilitate sustained IGF signaling. Elife 7, e32893 https://doi.org/10.7554/ elife.32893 (2018).

105. Li, J., Choi, E., Yu, H. \& Bai, X. C. Structural basis of the activation of type 1 insulin-like growth factor receptor. Nat. Commun. 10, 4567 (2019).

106. Ahmed, T. A. et al. SHP2 drives adaptive resistance to ERK signaling inhibition in molecularly defined subsets of ERK-dependent tumors. Cell Rep. 26(65-78), e65 (2019). 